\title{
QUEUING ISSUES OF BIG DATA ANALYTICS IN HEALTHCARE FRAME WORK
}

S. Maragathasundari

Assistant Professor, Department of Mathematics, Kalasalingam Academy of Research and Education, Krishnankovi, (India).

E-mail: maragatham01@gmail.com ORCID: https://orcid.org/0000-0003-1210-6411

G. Prabhu

Associate Professor, , Department of Mathematics, Kalasalingam Academy of Research and Education, Krishnankovil, (India).

E-mail: cprabhumath@gmail.com

ORCID: https://orcid.org/0000-0003-3879-3299

K. S. Dhanalakshmi

Assistant Professor, Department of Electronics and Communication Engineering,

Kalasalingam Academy of Research and Education

Krishnankovil, (India).

E-mail: k.s.dhanalakshmi@klu.ac.in

ORCID: https://orcid.org/0000-0001-6285-3656

Recepción: 11/11/2019 Aceptación: 03/12/2020 Publicación: 30/11/2021

\section{Citación sugerida:}

Maragathasundari, S., Prabhu, C., y Dhanalakshmi, K. S. (2021). Queuing issues of big data analytics in healthcare frame work. 3C Tecnología. Glosas de innovación aplicadas a la pyme, Edición Especial, (noviembre, 2021), 211-229. https://doi.org/10.17993/3ctecno.2021.specialissue8.211-229 


\section{ABSTRACT}

This paper explores an examination on Network traffic checking, investigation for upgrading system asset and improving client involvement in Healthcare Field. Be that as it may, existing systems accessible in medical clinics, which as a rule, depend on an elite server with expansive capacity limit, are not versatile for itemized examination of a vast volume of traffic information dependent on the patient examination subtleties, infection subtleties, blood bunch subtleties and the treatment given to them in intermittent premise and so on. The advantages of medicinal record trade (MRE), to give some examples, incorporate offering more data for doctor finding, giving better constant consideration to released patients, and dispensing with the misuse of copied examinations. In addition, the above system may be interrupted with all type of arriving patients, emergency cases, diagnosis period, consultation services etc. All the above issues happening inhuman services is drawn closer through Queuing hypothesis in this study. Queuing models are helpful for assessing the framework reaction time and all the execution proportions of the social insurance framework. Numerical delineation and an expand graphical investigation are completed toward the conclusion to approve the model. It gives a reasonable considered the calculated investigation of lining hypothesis in health insurance field.

\section{KEYWORDS}

Setup time, Single stage service, Compulsory Phase I Vacation, Optional Phase II Vacation, Restricted Admissibility. 


\section{INTRODUCTION}

Big data in wellbeing and science to handle the difficulties in new models is getting to be huge. In the period of enormous information, the most troublesome issues that stay to be comprehended are the means by which to proficiently manage large quantities and assortments of information. There are numerous diagnostic speculations and models. In this segment, late revelations in big data stockpiling and examination models are overviewed. The procurement of voluminous information relies upon an assortment of clients and gadgets, just as ground-breaking server farms to store and process the information. Therefore, building up an unrestricted system foundation is desperately required; this framework would make it conceivable to accumulate geographically circulated and quickly produced information and send them to server farms for end clients. In one investigation, members saw the different difficulties in Setting up such a system structure.

Among emergency clinics, Electronic medicinal record trade can give more data to doctor determination and diminish costs from copy examinations. The fast development of data and correspondence innovation has accelerated advancement of clinical data frameworks. An ever-increasing number of patient records, research facility reports, drug store drugs, and budgetary and medical coverage information are currently exchanged through PG systems. As of now, most emergency clinics have built up their very own electronic medicinal record frameworks. In any case, these frameworks just help singular medical clinics and don't give correspondence or offer assets among emergency clinics. Along these lines, it is troublesome for a patient to visit his specialist in one clinic and have his restorative record from another medical clinic accessible. The long-haul care units additionally can't get release data from the medical clinics. Every human services supplier must use much time and exertion to gather quiet data, along these lines causing information excess, and maybe notwithstanding imperiling patient wellbeing. 


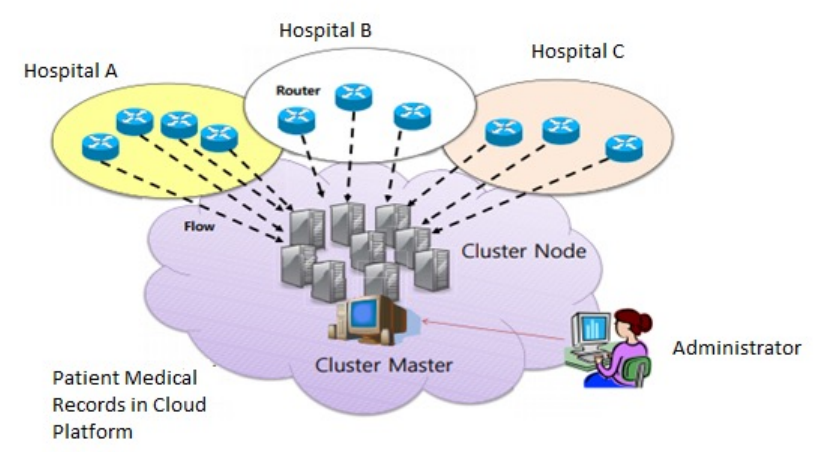

Graphic 1. Big Data Model in Healthcare System.

Source: own elaboration.

\subsection{QUEUING PROBLEM IN HEALTH CARE UNIT PROCESS}

Diverse applications have distinctive execution prerequisites. We list here seven such necessities:

1) Batch landing - Arrival of patients: Assume that the entry time of an outpatient at a clinic. Specifically, expanded asset use does not really suggest longer holding up neither lines nor longer patient stream times.

2) Set up time - Nursing Station: It is kept up by the doctor's training to look at the connection between inspecting room limit and patient stream crosswise over center-based execution measures. And furthermore distinguishing a few significant and generalizable parts of outpatient facility tasks

3) Single Stage Service - Fixed Normal Service: Traditionally, social insurance has been separated as either inpatient or outpatient. Inpatient care is given when patients are required to stay at a medical clinic or care office for the span of their treatment or disease. With outpatient social insurance, patients are dealt with and discharged that day. An arriving quiet check in and holds on to be called by a nursing room. Given both a nursing room and an inspecting room are accessible; the patient is brought to the nursing room. At the nursing room, the medical caretaker records the patient's indispensable insights and escorts the patient to an accessible looking at room. 
4) Feedback Service - "Visit Service": Owing to mechanical advances in diagnostics, prescriptions, and techniques, a greater part of social insurance needs is currently taken care of on an outpatient premise. The patient might be sent to the center research facility or x-beam office through the attendant helper station for doctor produced lab or x-beam work. This was regular amid physical examinations and wandering medical procedure.

5) Compulsory Phase I Vacation - Diagnosis period: The patient might be sent to the center lab or x-beam office by means of the attendant helper station for doctor produced lab or x-beam work. This was regular amid physical examinations and wandering medical procedure. During this season of Compulsory Phase I excursion the Server profit get-away (As long as the patient gets the records)

6) Optional Phase II Vacation: Final Consultation after conclusion the finish of a patient's "underlying" interview with their doctor was motioned by either the patient leaving the inspecting room (for the nursing station or registration work area) or the doctor leaving the looking at room. In the event that the underlying meeting finished with the doctor leaving the analyzing room, administration proceeded for the patient yet was not really performed in the patient's essence. For the most part, the expansion of administration as an "arrival" meeting included exercises that couldn't be finished inside the inspecting room.

7) Restricted Admissibility of Patient to the framework amid Optional get-away: Emergency/Accident Services - During this Optional Phase II get-away there will be a limited suitability of the patient to the framework is conceivable. "Administration" alludes to the period of time a patient went through in meeting with center work force while "stay" alludes to the all-out time spent at some area inside the facility. Pausing and administration times were effectively found as the contrast between the completion and beginning of two continuous administrations. Since the center lab and x-beam office are physically outside the family practice facility, just patient visits at these areas were recorded. 


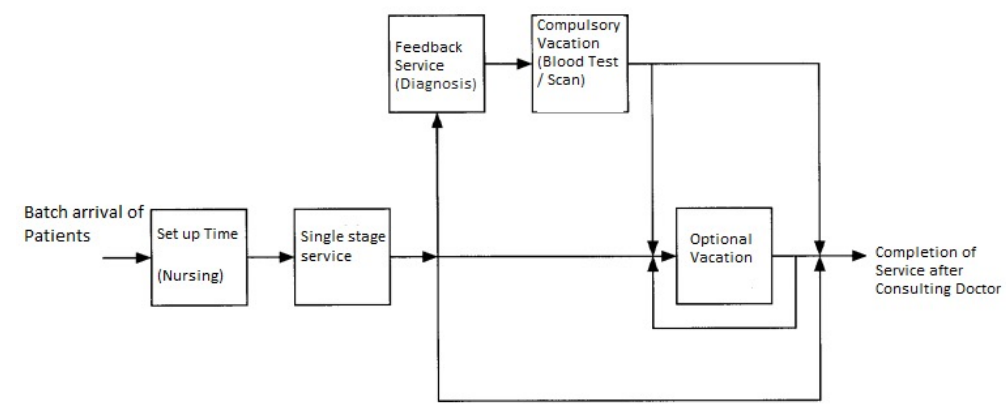

Graphic 2. Service Mechanism.

Source: own elaboration.

\section{METHODOLOGY}

\subsection{SOLVING OF HEALTH QUEUING PROBLEM BY VARIABLE SUPPLEMENTRY VARIABLE METHOD OF QUEUING THEORY}

The above process carried out in health care unit is a concrete problem which is well analyzed by queuing theory in this suggested current work. The queuing mechanism is developed based on the probability distribution in different range of communication. The above Queuing problem is solved through supplementary variable method of Queuing approach. Hence to start with, we describe the above queuing issue in terms of mathematical study of queuing theory. The above health care unit process is completely transformed to a queuing problem. Governing equations of the queuing problem are framed initially. Then the boundary conditions of the problem are explained. Then by the supplementary variable technique of usage of variable ' $z$ ', we derive the probability generating function of the queue size. Then by the tuberin property, length of the queue is found. Finally, the other execution measures of the problem are derived using Little's law. As clarified above in Health care unit process, the procedure comprises of arrival of patients, nurse station, normal fixed service, sojourn service, Diagnosis period, consultation period and emergency, accidental services. 


\subsection{THE HEALTH CARE UNIT QUEUING PROBLEM IS AS FOLLOWS IN TERMS OF MATHEMATICAL STUDY}

Client's arrival follows Poisson distribution. Administration pursues general circulation. To begin with, a set up time technique is presented in this procedure. Single phase of administration is given to all the arriving clients. On the off chance that any of the clients are in need, they are permitted to get an input administration, which gives a total attractive to all the arriving clients. After the fruition of the administration, the server needs to attempt an obligatory excursion, amid which to make the framework run easily to a more prominent degree, a total upkeep work to be done. Toward the finish of mandatory excursion, the server has the alternative to take an all-encompassing get-away if the submitted support work has not been finished amid the past get-away hour.

Restricted admissibility in the arriving customers is considered during the time of optional extended vacation to reduce the length of the queue. The lining issue (Health care unit enormous information issue) is all around explored by strengthening variable technique. For every one of the set-up time, administration process, mandatory get-away and discretionary broadened excursion, beneficial components are distinguished. A determined state line measure assignment and the distinctive execution checks like length of the line, number of clients in the framework, usage factor, latent time of the server, holding up time of the clients in the line likewise as in the structure are inferred. Numerical depiction legitimizes the model and the graphical outline gives a sensible picture about the decisions to be taken before the beginning of the affiliation. To break down the issue in social insurance unit, a verifiable endorsing is rendered close to the end, by strategies for looking numerical results and graphical examination of the model.

Huge information examination has been considered by various creators. In any case, the issue of huge information investigation in medicinal services unit is drawn closer through lining hypothesis is another thought of execution in this examination. McAfee and Brynjolfsson (2012) examined the work on big information the board insurgency. Development of information investigation is totally very much concentrated by Lynch (2008). Big information pathologies are all around assessed by Jacobs (2009). 
Zikopoulos et al. (2011) made an analysis on examination for big business class Hadoop and Streaming Data. Manyika, Chui, and Brown (2011) learned about the developments and preparations on Big Data. Celi et al. (2013) examined the work on big information in the emergency unit. Sobhy et al. (2012) considered the enormous information on human services distributed computing system. Queuing frameworks with expansive scope of excursion arrangements have been planned by various authors. Single get-away strategy is all around examined by Choudhury (2002).

Dhanalakshmi and Maragathasundari (2018) contemplated Mobile ad hoc systems issue through queuing approach. Maragathasundari (2015) inferred the execution measures for a mass entry queuing model of three phases of administration with various excursion policies. Maragathasundari and Srinivasan (2012) made an Analysis on M/G/1 input line. Maragathasundari and Karthikeyan (2016) explored a mass queuing model with short and long get-away.

In this study, we have introduced the big data analytic issue as a batch arrival nonMarkovian queuing model with setup time, single service and feedback service. As this is a non markovian queuing model, service time follows a general distribution. A concept of Phase I compulsory vacation and phase II optional vacation are launched in this work which plays a very important role in our study. Here vacation in the sense we mean the maintenance work to be carried out in due course of time which helps the system to run smoothly to a maximum extent. It is expected that during the time of vacation, length of the queue will be maximized. So, to avoid the congestion in the system a phenomenon known to be restricted admissibility is brought in during the time of vacation.

\subsection{MATHEMATICAL DEPICTION OF THE MODEL}

The arithmetical interpretation of the Queuing framework has the capacity to be described by the resulting hypothesis:

Customers cluster arrival follows Poisson procedure Let $\lambda d_{i} d t(i=1,2,3 \ldots)$ be the first order probability where $0 \leq d_{i} \leq 1$ and $\sum_{i=1}^{n} d_{i}=1$ and $\lambda>0$ is the mean landing rate of the batches. There is one server giving single sort of administrations of general course. The 
organization time seeks after general (arbitrary) course with first basic distribution function $\mathrm{E}(\mathrm{x})$ and density function $\mathrm{e}(\mathrm{x})$. Let $\mu(\mathrm{x}) \mathrm{dx}$ be the prohibitive probability of organization finish of the principle period of organization in the midst of the interval $(x, x+d x)$, given that the snuck past time is $\mathrm{x}$, so that $\mu(x)=\frac{e(x)}{1-\bar{E}(x)}$

$$
e(x)=\mu(x) e^{-\int_{0}^{x} \mu(t) d t}
$$

For the purpose of maintenance work to be carried out, the server takes a compulsory vacation with probability $\mathrm{r}$.

For compulsory vacation, $\gamma_{a}(x)=\frac{\bar{a}(x)}{1-\bar{A}(a)(x)}$

$$
\bar{a}(x)=\gamma_{a}(x) e^{-\int_{0}^{x} \gamma_{a}(t) d t}
$$

After the completion of compulsory vacation, if in need the server may take an extended vacation with probability $\mathrm{k}$.

For the optional vacation, we have:

$$
\gamma_{b}(x)=\frac{\bar{b}(x)}{1-\bar{S}(b)(x)} \quad, \bar{b}(x)=\gamma_{b}(x) e^{-\int_{0}^{x} \gamma_{b}(t) d t}
$$

After completion of the service, if the client is disappointed with the essential administration, he can quickly join the tail of the first line as a feedback client with likelihood e to rehash the administration until the point when it is fruitful or may leave the framework with likelihood $1-\mathrm{e}$.

\subsection{GOVERNING EQUATIONS OF THE MODEL}

$S_{n}(t)$ - This is the probability that at time ' $t$ ', the server is in setup while there are ' $n$ ' customers in the queue. $(\mathrm{n} \geq 1)$

$$
\begin{aligned}
& \frac{d}{d t} S_{n}(t)+(\lambda+\gamma) S_{n}(t)=\lambda \sum_{i=1}^{n} c_{i} S_{n-i}(t)+\lambda c_{n} Q(t) . \\
& \frac{d}{d x} P_{n}(x)+(\lambda+\eta(x)) P_{n}(x)=\lambda \sum_{i=1}^{n} c_{i} P_{n-i}(x) \\
& \frac{d}{d x} P_{0}(x)+(\lambda+\eta(x)) P_{0}(x)=0 . \\
& \frac{d}{d x} V_{n}^{(a)}(x)+\left(\lambda+\gamma_{a}(x)\right) V_{n}^{(a)}(x)=\lambda \sum_{i=1}^{n} c_{i} v_{n-i}^{(a)}(x), n \geq 1
\end{aligned}
$$




$$
\begin{aligned}
& \frac{d}{d x} V_{0}^{(a)}(x)+\left(\lambda+\gamma_{a}(x)\right) V_{0}^{(a)}(x)=0 \\
& \frac{d}{d x} V_{n}^{(b)}(x)+\left(\lambda+\gamma_{b}(x)\right) V_{n}^{(b)}(x)=\lambda(1-\beta) V_{n}^{(b)}(x)+\lambda \beta \sum_{i=1}^{n} c_{i} v_{n-i}^{(b)}(x), n \geq 1 \\
& \frac{d}{d x} V_{0}^{(b)}(x)+\left(\lambda+\gamma_{b}(x)\right) V_{0}^{(b)}(x)=\lambda(1-\beta) V_{0}^{(b)}(x) \\
& \lambda Q=\int_{0}^{\infty} V_{0}^{(\beta)}(x) \gamma_{b}(x) d x+(1-k) \int_{0}^{\infty} V_{0}^{(a)}(x) \gamma_{a}(x) d x+e \int_{0}^{\infty} p_{0}(x) \gamma(x) d x
\end{aligned}
$$

The accompanying limit conditions are utilized to settle the above conditions.

$$
\begin{aligned}
& p_{n}(0)=(1-k) \int_{0}^{\infty} V_{n+1}^{(a)}(x) \gamma_{a}(x) d x+\int_{0}^{\infty} V_{n+1}^{(b)}(x) \gamma_{b}(x) d x+e \int_{0}^{\infty} p_{n}(x) \eta(x) d x+\lambda c_{n+1} Q . \\
& V_{n}^{(a)}(0)=r(1-e) \int_{0}^{\infty} p_{n}(x) \eta(x) d x+r e \int_{0}^{\infty} p_{n-1}(x) \eta(x) d x . \\
& V_{n}^{(b)}(0)=k \int_{0}^{\infty} V_{n}^{(a)}(x) \gamma_{a}(x) d x .
\end{aligned}
$$

\section{DISTRIBUTION OF THE QUEUE LENGTH}

To settle conditions (1) to (7) for a shut structure arrangement we pursue the system set out beneath:

We multiply (1) by $z^{n}$ and sum over $n$ from 1 to $\infty$ add it to (2), we get

$$
\begin{aligned}
& (\lambda-\lambda c(z)+\gamma) s_{q}(z)=\lambda Q c(z) . \\
& \frac{d}{d x} p_{q}(x, z)+(\lambda-\lambda c(z)+\eta(x)) p_{q}(x, z)=0 . \\
& \frac{d}{d x} V_{q}^{(a)}(x, z)+\left(\lambda-\lambda c(z)+\gamma_{a}(x)\right) V_{q}^{(a)}(x, z)=0 . \\
& \frac{d}{d x} V_{q}^{(b)}(x, z)+\left(\lambda \beta-\lambda \beta c(z)+\gamma_{b}(x)\right) V_{q}^{(b)}(x, z)=0 .
\end{aligned}
$$

Multiplying (9) by $z^{\mathrm{n}+1}$ and summing over $n$ from 0 to $\infty$ results in:

$$
\begin{aligned}
& z p_{q}(0, z)=(1-k) \int_{0}^{\infty} V_{q}^{(a)}(x, z) \gamma_{a}(x) d x+\int_{0}^{\infty} V_{q}^{(b)}(x, z) \gamma_{b}(x) d x+e \int_{0}^{\infty} p_{q}(x, z) \eta(x) d x+ \\
& \lambda c(x) Q-\left[e \int_{0}^{\infty} p_{0}(x, z) \eta(x) d x+(1-k) \int_{0}^{\infty} V_{0}^{(a)}(x, z) \gamma_{a}(x) d x+\int_{0}^{\infty} V_{0}^{(b)}(x, z) \gamma_{b}(x) d x\right] .
\end{aligned}
$$

Using (8) in (16), we get:

$$
\begin{aligned}
& z p_{q}(0, z)=(1-k) \int_{0}^{\infty} V_{q}^{(a)}(x, z) \gamma_{a}(x) d x+\int_{0}^{\infty} V_{q}^{(b)}(x, z) \gamma_{b}(x) d x+e \int_{0}^{\infty} p_{q}(x, z) \eta(x) d x+ \\
& \lambda(c(z)-1) Q .
\end{aligned}
$$




$$
\begin{aligned}
& V_{q}^{(a)}(0, z)=r(1-e) \int_{0}^{\infty} p_{q}(x, z) \eta(x) d x+\operatorname{rez} \int_{0}^{\infty} p_{q}(x, z) \eta(x) d x . \\
& V_{q}^{(b)}(0, z)=k \int_{0}^{\infty} V_{q}^{(a)}(x, z) \gamma_{a}(x) d x .
\end{aligned}
$$

Now integrating (13) from 0 to $x$ yields,

$$
p_{q}(x, z)=p_{q}(0, z) e^{-(\lambda-\lambda c(z))-\int_{0}^{x} \eta(t) d t} .
$$

Integrating (20) by parts with respect to $X$ yields,

$$
p_{q}(z)=p_{q}(0, z)\left(\frac{1-\bar{E}(a)}{a}\right), a=\lambda-\lambda c(z) .
$$

Where, $\bar{E}(a)=\int_{0}^{\infty} e^{-(\lambda-\lambda c(z)) x} d E(x)$ is the Laplace stieltjes transform of the service times $E(\mathrm{x})$.

Multiply (20) by $\eta(\mathrm{x})$ on both the sides, we get

$$
\int_{0}^{\infty} p_{q}(x, z) \eta(x) d x=p_{q}(0, z) \bar{E}(a)
$$

Using (20) in (18), we get

$$
V_{q}^{(a)}(0, z)=[r-r e(1+z)] p_{q}(0, z) \bar{E}(a)
$$

Similarly,

$$
\begin{gathered}
\int_{0}^{\infty} V_{q}^{(a)}(z)=V_{q}^{(a)}(0, z)\left(\frac{1-\bar{A}(a)}{a}\right) \\
=[r-r e(1+z)] p_{q}(0, z) \bar{E}(a)\left[\frac{1-\bar{A}(a)}{a}\right] . \\
\int_{0}^{\infty} V_{q}^{(a)}(x, z) \gamma_{a}(x) d x=[r-r e(1+z)] p_{q}(0, z) \bar{E}(a) \bar{A}(a) .
\end{gathered}
$$

Also,

$$
V_{q}^{(b)}(z)=k[r-r e(1+z)] p_{q}(0, z) \bar{E}(a) \bar{A}(a)\left[\frac{1-\bar{S}(b)}{b}\right], b=\lambda b-\lambda b c(z) .
$$

Hence,

$$
\int_{0}^{\infty} V_{q}^{(b)}(x, z) \gamma_{b}(x) d x=k[r-r e(1+z)] p_{q}(0, z) \bar{E}(a) \bar{A}(a) \bar{S}(b)
$$


Now using (22), (25) and (27) in (17), we get

$$
\begin{aligned}
& z p_{q}(0, z)=(1-k)[r-r e(1+z)] p_{q}(0, z) \bar{E}(a) \bar{A}(a) \\
& \quad+k[r-r e(1+z)] p_{q}(0, z) \bar{E}(a) \bar{A}(a) \bar{S}(b)+e p_{q}(0, z) \bar{E}(a)+\lambda(c(z)-1) Q \\
& \Rightarrow p_{q}(0, z)=\frac{\lambda(c(z)-1) Q}{z-e \bar{E}(a)-[r-r e(1+z)] \bar{E}(a) \bar{A}(a)(1-k+k \bar{S}(b))} .
\end{aligned}
$$

Substituting (28) in (21), (24) and (26), we get

$$
\begin{aligned}
& p_{q}(z)=\frac{-(1-\bar{E}(a)) Q}{z-e \bar{E}(a)-[r-r e(1+z)] \bar{E}(a) \bar{A}(a)(1-k+k \bar{S}(b))} . \\
& V_{q}^{(a)}(z)=\frac{-[r-r e(1+z)] \bar{E}(a) \bar{A}(a) Q}{z-e \bar{E}(a)-[r-r e(1+z)] \bar{E}(a) \bar{A}(a)(1-k+k \bar{S}(b))} . \\
& V_{q}^{(b)}(z)=\frac{-[r-r e(1+z)] \bar{E}(a) \bar{A}(a) \bar{S}(b) Q}{\beta(z-e \bar{E}(a)-[r-r e(1+z)] \bar{E}(a) \bar{A}(a)(1-k+k \bar{S}(b)))} .
\end{aligned}
$$

Also,

$S_{q}(z)=\frac{\lambda Q c(z)}{\lambda-\lambda c(z)+\gamma}$

\section{EXECUTION MEASURES}

\section{(i) LIKELIHOOD GENERATING CAPACITY OF THE QUEUE SIZE}

Let $D_{q}^{*}(z)$ be the probability generating function of the queue size

$$
D_{q}^{*}(z)=S_{q}(z)+P_{q}(z)+V_{q}^{(a)}(z)+V_{q}^{(b)}(z)
$$

Adding (29) - (31), we get

$$
D_{q}^{*}(z) \quad=\frac{Q[\beta \lambda C(z)]\left[\begin{array}{c}
\beta[z-e \bar{E}(a)-(r-r e(1+z)) \bar{E}(a) \bar{A}(a)(1-k+k \bar{S}(b))]-(\lambda-\lambda C(z)+\gamma) \\
{[[\beta(1-\bar{E}(a) Q+(r-r e(1+z)) \bar{E}(a) \bar{A}(a) Q(\beta+\bar{S}(b)))]]}
\end{array}\right]}{(\lambda-\lambda C(z)+\gamma) \beta[[z-e \bar{E}(a)-(r-r e(1+z)) \bar{E}(a) \bar{A}(a)(1-k+k \bar{S}(b))]]} .
$$

The idle time $Q$ is determined using the normalization condition $D_{q}^{*}(1)+Q=1$.

From, the utilization factor $\rho$ can be determined. 


\section{(ii) STEADY STATE ARRANGEMENT OF THE QUEUE SIZE}

Let $L_{q}$ a chance to demonstrate the reliable state typical number of customers in the line. By then

$$
L_{q}=\left.\frac{d}{d z} D_{q}^{*}(z)\right|_{z=1}=\left.\frac{d}{d z}\left\{\frac{N(Z)}{D(Z)}\right\}\right|_{z=1}
$$

Where $N(Z)$ and $D(Z)$ are the numerator and denominator of (27).

Since $D_{q}^{*}(z)=\frac{0}{0}$ at $=1$, we utilize two-fold separation and get

$$
\begin{aligned}
& L_{q}=\lim _{z \rightarrow 1} \frac{d}{d z} D_{q}^{*}(z)=\frac{D^{\prime}(1) N^{\prime \prime}(1)-D^{\prime \prime}(1) N^{\prime}(1)}{2\left(D^{\prime}(1)\right)^{2}} . \\
& D^{\prime}(1)=\beta\{-\lambda[1-e-(r-2 r e)]+\gamma[1-e \lambda E(k)-[-r e+(r-2 r e) \lambda(E(k)+E(A)+ \\
& \beta k E(s))]]\} . \\
& D^{\prime \prime}(1)=\beta\{-\lambda[-r e+(r-2 r e) \lambda(E(k)+E(A)+\beta k E(s))]\}+(-\lambda)[1-e \lambda E(k)-[-r e+ \\
& (r-2 r e) \lambda \lambda(E(k)+E(A)+\beta k E(s))]]+\gamma\left[-e \lambda^{2} E\left(k^{2}\right)-r e \lambda(E(k)+E(A)+\beta k E(s))-\right. \\
& r e \lambda(E(k)+E(A)+\beta k E(s))+(r-2 r e) \lambda^{2}\left[E\left(k^{2}\right)+E\left(A^{2}\right)+k \beta E\left(s^{2}\right)+2 E(k) E(A)+\right. \\
& 2 k \beta E(s)(E(k)+E(A))]] . \\
& N^{\prime \prime}(1)=(\beta \lambda+1)\{1-e \lambda E(k)+r e-\lambda(r-2 r e)(E(k)+E(A)+\beta k E(s))\}+ \\
& \beta \lambda\left\{-e \lambda^{2} E\left(k^{2}\right)+r e \lambda(E(k)+E(A)+\beta k E(s))+r e(E(k)+E(A)+\beta k E(s))+(r-\right. \\
& 2 r e)\left[-\lambda E\left(A^{2}\right)-2 \lambda E(k) E(A)-2 E(A) k \beta E(s) \lambda-\lambda E\left(A^{2}\right)-\lambda E(k) k \beta E(s)-\lambda E(A) k \beta E(s)-\right. \\
& \left.\left.\lambda E(k) E(s) k \beta-\lambda k \beta^{2} E\left(s^{2}\right)\right]\right\}-\lambda(r-2 r e) \beta-\lambda(-\lambda E(A)-r e \beta+\beta(r-2 r e)(E(k)+ \\
& E(A)+\beta k E(s)))+\gamma\left[-\lambda^{2} E\left(k^{2}\right)-r e \lambda \beta(E(k)+E(A)+E(s))-r e \lambda(E(k)+E(A)+\right. \\
& \beta E(s))+(r-2 r e)\left(-\lambda \beta E\left(k^{2}\right)-\lambda \beta E\left(A^{2}\right)-2 \lambda E(k) E(A) \beta-\lambda \beta E(s)[E(k)+E(A)]-\right. \\
& \left.\left.\lambda \beta^{2} E\left(s^{2}\right)\right)\right] . \\
& N^{\prime}=\beta \lambda[1-e-(r-2 r e)]+[1-e \lambda E(k)-[-r e+(r-2 r e)(E(k)+E(A)+k \beta E(s))] .
\end{aligned}
$$

Substituting (34) - (37) in (23), we obtain $L_{q}$ and all the other measures are obtained using

Little's formula

$$
\text { (iii) } W_{q}=\frac{L_{q}}{\lambda}, W=\frac{L}{\lambda}, L=L_{q}+\rho \text {. }
$$




\section{NUMERICAL RESULTS}

We depict a numerical perspective with a definitive goal to see the impact and credibility of our consequences of the specific parameters utilized in our model. The estimations of the parameters are collected with a definitive target that the enduring condition is not disturbed.

Assume service time follows an exponential distribution:

$$
\begin{gathered}
E(k)=\frac{1}{\eta}, E\left(k^{2}\right)=\frac{2}{\eta^{2}}, E(A)=\frac{1}{\gamma_{a}}, E\left(A^{2}\right)=\frac{2}{\gamma_{a}{ }^{2}}, E(s)=\frac{1}{\gamma_{b}}, E\left(s^{2}\right)=\frac{2}{\gamma_{b}{ }^{2}} \gamma=0.8, \eta=3, \lambda \\
=4, \beta=1.5, \gamma_{a}=2.5, \gamma_{b}=3.5, r=0.5,
\end{gathered}
$$

Table 1. Effect of change ofe $(e=6,5.5,5,4.5,4)$.

\begin{tabular}{|c|c|c|c|c|c|}
\hline $\boldsymbol{Q}$ & $\boldsymbol{\rho}$ & $\boldsymbol{L q}$ & $\boldsymbol{L}$ & $\boldsymbol{W}_{\boldsymbol{Q}}$ & $\boldsymbol{W}$ \\
\hline 0.7239 & 0.2761 & 15.551 & 15.827 & 5.184 & 5.184 \\
\hline 0.7512 & 0.2488 & 15.167 & 15.415 & 5.056 & 5.139 \\
\hline 0.7728 & 0.2272 & 14.889 & 15.116 & 4.963 & 5.039 \\
\hline 0.7903 & 0.2097 & 14.679 & 14.889 & 4.893 & 4.963 \\
\hline 0.8049 & 0.1951 & 14.518 & 14.713 & 4.839 & 4.904 \\
\hline
\end{tabular}

Source: own elaboration.

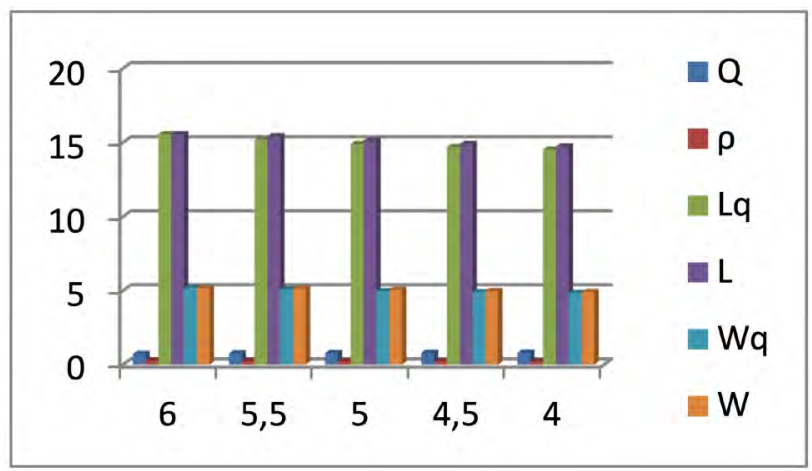

Graphic 3. Effect of Variation of $e$.

Source: own elaboration. 
Table 2. Effect of Variation Of $\gamma(\gamma=2.1,2.2,2.3,2.4,2.5)$

\begin{tabular}{|c|c|c|c|c|c|}
\hline $\boldsymbol{Q}$ & $\boldsymbol{\rho}$ & $\boldsymbol{L q}$ & $\boldsymbol{L}$ & $\mathbf{W}$ \\
\hline 0.8751 & 0.1249 & 4.9967 & 5.1216 & 1.2492 & 1.2804 \\
\hline 0.8806 & 0.1194 & 4.3960 & 4.5154 & 1.0990 & 1.1289 \\
\hline 0.8856 & 0.1144 & 3.9666 & 4.0810 & 0.9916 & 1.0202 \\
\hline 0.8902 & 0.1098 & 3.5038 & 3.6136 & 0.8759 & 0.9034 \\
\hline 0.8945 & 0.1055 & 3.0698 & 3.1753 & 0.7674 \\
\hline
\end{tabular}

Source: own elaboration.

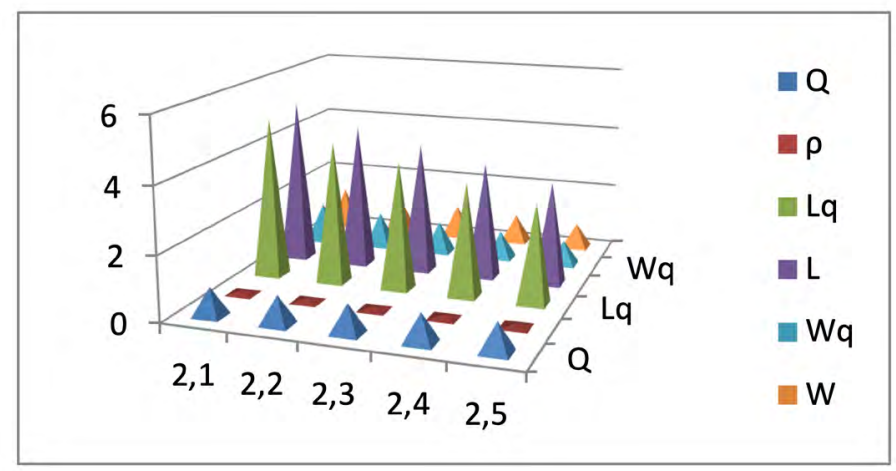

Graphic 4. Effect of Variation of $\gamma$.

Source: own elaboration.

Table 3. Effect of Variation Of $\beta(\beta=1.6,1.8,2,2.2,2.4)$

\begin{tabular}{|c|c|c|c|c|c|}
\hline $\boldsymbol{Q}$ & $\rho$ & $L q$ & $L$ & $W_{Q}$ & $W$ \\
\hline 0.6952 & 0.3048 & 20.334 & 20.639 & 5.083 & 5.1596 \\
\hline 0.7077 & 0.2923 & 19.597 & 19.889 & 4.899 & 4.9723 \\
\hline 0.7190 & 0.2810 & 19.047 & 19.328 & 4.762 & 4.8319 \\
\hline 0.7295 & 0.2705 & 18.632 & 18.902 & 4.657 & 4.7255 \\
\hline 0.7391 & 0.2609 & 18.319 & 18.579 & 4.579 & 4.6450 \\
\hline
\end{tabular}

Source: own elaboration. 


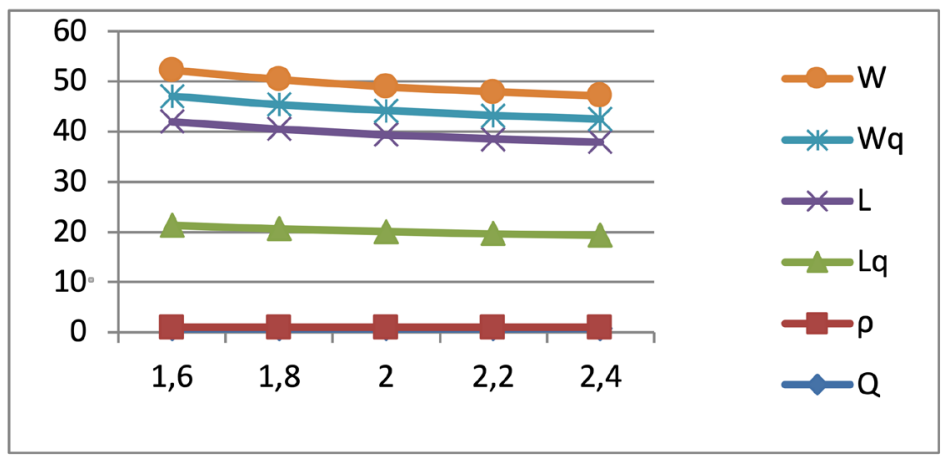

Graphic 5. Effect of Variation of $\beta$.

Source: own elaboration.

\section{DISCUSSION}

It is evident from Table 1 that lessening the estimation of e, decreases the traffic power, normal length of the line and the normal reaction time while the server inert time increases. From Table 2, the information we collect here is, as the probability of set up time constructs, it prompts a smart presentation of the structure. From now on, the length of the line gets lessens. Also, the other execution measures in like manner get decreased. Furthermore, restricted admissibility yin table 3 expect a noteworthy activity in this model. To avoid stop up this thought is exhibited. It means a nice impact in the model. On account of the extension in the confined suitability, the blockage in the structure is avoided. Length of the line gets reduced and moreover the holding up time of the customers in like manner gets diminished. All of the examples showed up by this are true to form. In extension, Graphical examination gives an undeniable picture about the model described in this paper.

\section{CONCLUSIONS}

In this model, we analyzed a Queuing framework with general administration conveyance with various vacation arrangements. Here two kinds of vacations are presented viz. mandatory excursion and discretionary broadened get-away. Also, the idea of feedback administration encourages the clients to get a total fulfillment regarding the administration. Amid the season of both the excursions, a legitimate support work is done which encourages the framework to run easily without intrusion to a greatest dimension. By including another presumption, restricted admissibility in this model, an assorted and a much-created lining 
framework is developed. The relentless state arrangement and the execution proportions of the lining framework are determined. As a future work, building up the above lining model with administration intrusion, postpone time, shut down time, Balking and reneging is proposed

\section{REFERENCES}

Celi, L. A., Mark, R. G., Stone, D. J., \& Montgomery R. A. (2013). 'Big data' in the intensive care unit: closing the data loop. American fournal of Respiratory and Critical Care Medicine. 187(11), 1157-1160. https://doi.org/10.1164/rccm.201212-2311ed

Choudhury, G. (2002). A batch arrival queue with a vacation time under single vacation policy. Computers and Operations Research, 29(14), 1941-1955. https://doi.org/10.1016/ S0305-0548(01)00059-4

Dhanalakshmi, K. S., \& Maragathasundari, S. (2018). Mobile ad hoc networks problem- A queuing approach. Internationaljournal of communication networks and distributed systems, 21(4). https://www.inderscience.com/info/inarticle.php?artid=95363

Jacobs, A. (2009). The pathologies of big data. Communications of the ACM, 52(8), 36-44. https://doi.org/10.1145/1536616.1536632

Lynch, G. (2008). Big data: how do your data grow? Nature. 455(7209), 28-29. https://doi. org/10.1038/455028a

Manyika,J., Chui, M., \& Brown, B. (2011). "Big Data" The Next Frontier for Innovation, Competition, and Productivity. McKinsey Global Institute.

Maragathasundari, S. (2015). A bulk arrival queuing model of three stages of service with different vacation policies, service interruption and delay time. American International Journal of Research in Science, Technology, Engineering $\mathcal{E}^{2}$ Mathematics, 11(1), 52-56. https:/ / www.researchgate.net/publication/295605742_A_bulk_arrival_queueing_model_ of_three_stages_of_service_with_different_vacation_policies_service_interruption_ and_delay_time 
Maragathasundari, S., \& Karthikeyan, K. (2016). A bulk queuing model of optional second phase service with short and long vacations. International fournal of scientific research in science and technology, 2(5), 196-201. https://www.researchgate.net/ publication/308917899_A_Bulk_Queuing_Model_of_Optional_Second_Phase_ Service_with_Short_and_Long_Vacations

Maragathasundari, S., \& Srinivasan, S. (2012). Analysis of M/G/1 feedback queue with three stages and multiple server vacation. Applied mathematical sciences, 6(125), 6221-6240. https://www.researchgate.net/publication/259962534_Analysis_of_ MG1_Feedback_Queue_with_Three_Stage_and_Multiple_Server_Vacation

McAfee, A., \& Brynjolfsson, E. (2012). Big data: the management revolution. Harvard Business Reviewe, 90(10), 60-68. https://www.researchgate.net/ publication/232279314_Big_Data_The_Management_Revolution

Sobhy, D., El-Sonbaty, Y., AbouElnasr, M., \& MedGloud. (2012). Healthcare cloud computing system. Proceedings of the International Conference for Internet Technology and Secured Transactions; London, UK. IEEE (pp. 161-166).

Zikopoulos, P., Eaton, G., deRoos, D., Deutsch, T., \& Lapis, G. (201 1). Understanding Big Data: Analytics for Enterprise Class Hadoop and Streaming Data. McGraw-Hill Osborne Media. 
